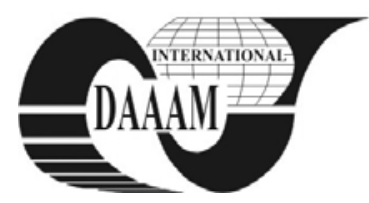

Annals of DAAAM for 2011 \& Proceedings of the 22nd International DAAAM Symposium, Volume 22, No. 1, ISSN 1726-9679 ISBN 978-3-901509-83-4, Editor B. Katalinic, Published by DAAAM International, Vienna, Austria, EU, 2011 Make Harmony between Technology and Nature, and Your Mind will Fly Free as a Bird

\title{
ROBUST DESIGN FOR PI CONTROLLERS: AN ALGEBRAIC APPROACH
}

\author{
SURYNEK, T[omas] \& PROKOP, R[oman]
}

\begin{abstract}
The main purpose of this contribution is to present a nontraditional, innovative approach of controller design for first order linear systems with parametric uncertainty. A special task to find a fixed PI controller stabilizing simultaneously stable and unstable controlled system is solved. So, the control task is aimed to a class of robust control principles. The controller design is performed in the ring of proper and stable rational functions $R_{p s}$ by means of algebraic tools. A set of stabilizing controllers are given by a solution of Diophantine equation in this ring. This approach offers $a$ scalar positive parameter $m>0$ which influences stability and behavior of the proposed closed loop system. The methodology is illustrated by an example and a stability analysis is outlined. Simulations are performed in the Matlab environment.

Key words: controller design, PI controller, algebraic approach, Diophantine equations, parametric uncertainty
\end{abstract}

\section{INTRODUCTION}

The most effective way how to change the stability properties of a given system is to exploit a feedback loop. Moreover, in industrial applications the most frequent feedback controller is of a PI or PID type, see (Åström \& Hägglund, 1995). Also, hundreds of principles and methods for PI and PID tuning have been developed many of them are reffered in (O'Dwyer, 2009). In this field, penetrating of algebraic tools has been visible for decades, see (Kučera, 1993), (Prokop \& Corriou, 1997). The traditional approach in algebraic design utilizes the ring of polynomials. However, polynomials have some drawbacks for robust control philosophy. Polynomial problems can be overcome by introducing the ring of proper and stable rational functions $R_{p s}$ introduced by (Vidyasagar, 1985).

The main aim of the contribution is to solve a special task. A first order system with a parametric uncertainty is considered. The task is to find a PI controller with fixed parameters so that the feedback system is stable for all possible combinations of system parameters. The solution is performed in the ring $R_{p s}$ by a general solution of a stabilizing Diophantine equation.

\section{SYSTEM DESCRIPTION}

The traditional approach for description of continuous-time linear systems uses transfer functions as a ratio of two polynomials. The reformulation for the ring $R_{p s}$ is quite simple and it can be expressed by the following relation:

$$
\begin{gathered}
H(s)=\frac{b(s)}{a(s)}=\frac{b(s)}{(s+m)^{n}} / \frac{a(s)}{(s+m)^{n}}=\frac{B(s)}{A(s)} \\
m>0, n=\max \{\operatorname{deg} a(s), \operatorname{deg} b(s)\}
\end{gathered}
$$

Simply speaking, the denominator and numerator must be divided by the same stable polynomial, see (Prokop \& Corriou, 1997) for details. The scalar positive parameter $m>0$ can be also conveniently used as a tuning knob for control behavior.

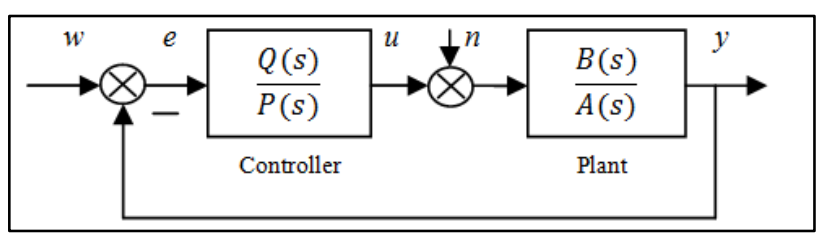

Fig. 1. General closed-loop system configuration

A traditional feedback system is depicted in Fig. 1. The fundamental and basic task of every feedback system is to ensure stability. One of the important algebraic results for control theory (Vidyasagar, 1985) is that the stability of feedback configuration in Fig. 1 is ensured by all solutions of Diophantine equation

$$
A P+B Q=1
$$

Under the condition of coprimeness of $A, B$ there exist an infinite number of pairs $P, Q$ fulfilling (2) in the form

$$
Q=Q_{0}-A T, P=P_{0}+B T
$$

where $P_{0}, Q_{0}$ are some particular solutions of (2) and $T$ is arbitrary element of the ring $R_{p s}$ fulfilling $P_{0}+B T \neq 0$. Probably, the second most important task of a feedback system is to ensure reference tracking. According to Fig. 1, a reference signal and load disturbance can be also expressed in $R_{p s}$

$$
w(s)=\frac{G_{w}}{F_{w}}, n(s)=\frac{G_{n}}{F_{n}}
$$

Also, further algebraic consequence for the error $(n(s)=0)$

$$
e(s)=\frac{A P}{A P+B Q} W(s)
$$

is that the denominator of (4) $F_{w}$ divides the denominator of the controller $P$. Details can be found e.g. in (Vidyasagar, 1985), (Kučera, 1993).

\section{PI CONTROLLER DESIGN}

A first order system and a stepwise reference can be expressed as fractions in $R_{p s}$ by the following relations:

$$
G(s)=\frac{b_{0}}{(s+m)} / \frac{s+a_{0}}{(s+m)}, W(s)=\frac{1}{(s+m)} / \frac{s}{(s+m)}
$$


Firstly, a particular solution of (2) must be found. Equation (2) takes the form

$$
\frac{s+a_{0}}{s+m} p_{0}+\frac{b_{0}}{s+m} q_{0}=1
$$

Clearly, a pair $p_{0}, q_{0}$ is obtained by comparison of appropriate coefficients of $s$ which results in

$$
p_{0}=1, \quad q_{0}=\frac{m-a_{0} p_{0}}{b_{0}}
$$

Now, from all solutions (3) must be chosen a pair $P, Q$ with the required divisibility condition $F_{w} \backslash P$. The derivation can be found in (Prokop \& Corriou 1997) and it results in PI controller in the form

$$
P=\frac{s}{s+m}, Q=\frac{\frac{2 m-a_{0}}{b_{0}} s+\frac{m^{2}}{b_{0}}}{s+m}
$$

Then the final transfer function of the controller is

$$
G_{c}(s)=\frac{\tilde{q}_{1} s+\tilde{q}_{0}}{s}, \text { where } \quad \tilde{q}_{1}=\frac{2 m-a_{0}}{b_{0}}, \quad \tilde{q}_{0}=\frac{m^{2}}{b_{0}}
$$

Notice that both controller parameters are functions of the tuning parameter $m$, generally nonlinear.

\section{EXAMPLE AND ANALYSIS}

Suppose a first order system with interval uncertainty in denominator

$$
G(s)=\frac{5}{s+a_{0}} \quad, \quad a_{0} \in<-0.5 ; 1>
$$

It is obvious that for a part of the uncertainty interval the system is stable and for a rest of the interval is unstable. For the robust control design it is necessary to choose a nominal plant. Usually, nominal plant parameters are chosen from the middle of the interval uncertainty, i.e. $\tilde{a}_{0}=0.25$ in this example. Trasfer function (11) is naturally considered in $R_{p s}$ in form (6). According to the mentioned methodology, the controller for the nominal plant was derived in the form of (10). For any plant parameter $a_{0}$, the characteristic polynomial $A P+B Q$ of the closed-loop takes the form

$$
s^{2}+\left(2 m+a_{0}-\tilde{a}_{0}\right) s+m^{2}
$$

Necessary and sufficient stability condition for (12) is that all coefficients in (12) must be positive $\left(2 m+a_{0}-\tilde{a}_{0}>0\right)$. This condition for the all potential values of $a_{0} \in<-0.5 ; 1>$ leads to the condition

$$
m>0.375
$$

\section{SIMULATION RESULTS}

Simulations were performed in Matlab Simulink. The stepwise reference is changed in the time $t=50 \mathrm{~s}$ and the load disturbance is applied at the time $t=100 \mathrm{~s}$. The nominal control behavior is depicted in Fig. 2. It means that nominal plant $\left(\tilde{a}_{0}=0.25\right)$ is controlled by nominal controller (10) for the same values of $m$. Fig. 3 illustrates the control responses for the boundary value $a_{0}=1.0$ for two values of $m=0.3, m=0.6$. In both cases the responses are stable. The different situation occurs for the second border value of $a_{0}=-0.5$. The stable response is only for the tuning parameter $m=0.6$ while the value $m=0.3$ represents unstable behavior since this value contradicts with condition (13).

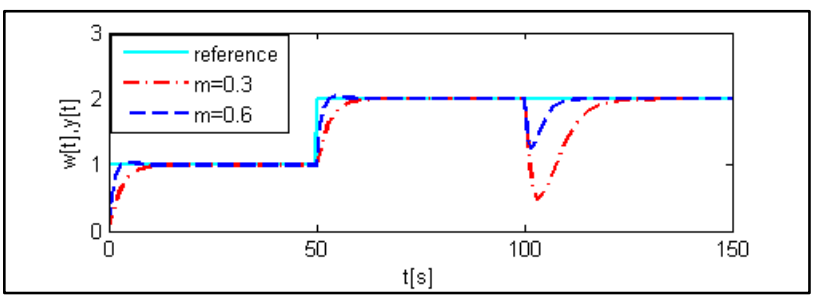

Fig. 2. Control response for $\tilde{a}_{0}=0.25$

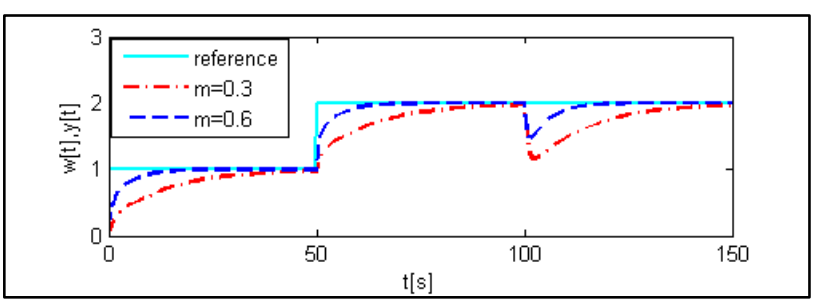

Fig. 3. Control responses for $a_{0}=1$

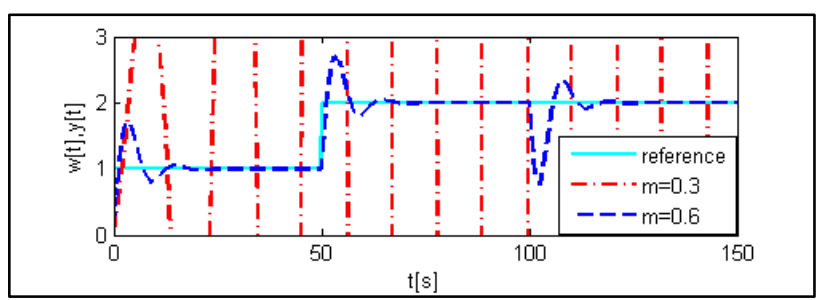

Fig. 4. Control response for $a_{0}=-0.5$

\section{CONCLUSION}

A design method for control of system with parametric uncertainty was introduced. The proposed methodology is based on solving Diophantine equation in the ring $R_{p s}$. A first order controlled plant generates a class of PI controllers. Analysis of robustness and stability is outlined. Simulation results of an illustrative example confirm transparent performance and applicability of the presented approach. The methodology can be easily generalized for higher order systems and for multiple uncertainty intervals.

\section{ACKNOWLEDGEMENTS}

The work behind the article was supported by the internal grant agency of Tomas Bata University in Zlin with NO. IGA/36/FAI/11/D and by the European Regional Development Fund under the project CEBIA-Tech No. CZ.11.05./2.1.00 /03.0089.

\section{REFERENCES}

Åström, K.J. and T. Hägglund (1995). PID Controllers: Theory, Design and Tuning, Instrument Society of America, USA

Kučera, V. (1993). Diophantine equations in control - A survey, Automatica, Vol. 29, No.6, pp. 1361-1375

O’Dwyer, A. (2009). Handbook of PI and PID Controller Tuning Rules, Imperial College Press

Prokop, R. and J.P. Corriou (1997). Design and analysis of simple robust controllers, Int. J. Control , Vol. 66, No. 6, pp. 905-921

Vidyasagar, M. (1985). Control system synthesis: a factorization approach, MIT Press, Cambridge, M.A. 